\title{
Charge storage and electron/light emission properties of silicon nanocrystals
}

\author{
S. Oda*, S.Y. Huang, M.A. Salem, D. Hippo, H. Mizuta \\ Quantum Nanoelectronics Research Center and Department of Physical Electronics, Tokyo Institute of Technology, Tokyo 152-8552, Japan
}

Available online 31 December 2006

\begin{abstract}
Monodispersed silicon nanocrystals show novel electrical and optical characteristics of silicon quantum dots, such as single-electron tunneling, ballistic electron transport, visible photoluminescence and high-efficiency electron emission.

Single-electron memory effects have been studied using a short-channel MOSFET incorporating Si quantum dots as a floating gate. Surface nitridation of Si nanocrystal memory nodes extends the charge-retention time significantly. Single-electron storage in individual Si dots has been evaluated by Kelvin probe force microscopy.

Photoluminescence and electron emission are observed for surface-oxidized silicon nanocrystals. Efficiency of the no-phonon-assisted transition increases with decreasing core Si size. Electron emission efficiency as high as 5\% has been achieved for the Si-nanocrystalbased cold electron emitter devices. The non-Maxwellian energy distribution of emitted electrons suggests that the mechanism of electron emission is due to ballistic transport through arrays of surface-oxidized Si nanocrystals. Combined with the ballistic electron emission, the quasi-direct light emission properties can be used for developing Si-based lasers.
\end{abstract}

(C) 2007 Elsevier B.V. All rights reserved.

PACS: 73.22.-f; 73.23.Hk; 73.63.Bd; 78.67.Bf

Keywords: Silicon nanocrystals; Single electron memory devices; Photo luminescence

\section{Introduction}

The recent progress in fabrication technology of silicon nanostructures has made possible the observations of novel electrical and optical properties of silicon quantum dots, such as single-electron tunneling [1,2], ballistic transport [3], visible photoluminescence [4], and electron emission [5].

Nanocrystalline silicon (nc-Si) particles with size less than $10 \mathrm{~nm}$ were prepared by VHF plasma-enhanced decomposition of silane gas. Pulsed gas plasma processing, in which the nucleation and the growth period were controlled precisely, turned out to be effective for the preparation of monodispersed nc-Si particles [6].

Electrical properties of nc-Si particles were investigated by employing nanoscale electrodes, both planar and

\footnotetext{
${ }^{*}$ Corresponding author. Tel.: + 81357343048 ; fax: +81357343565

E-mail address: soda@pe.titech.ac.jp (S. Oda).
}

vertical configurations, prepared by electron-beam lithography. Coulomb blockade and Coulomb oscillations predominantly due to a single quantum dot were readily modeled as also the interactions of electrons between neighboring dots $[7,8]$. Quantized conductance due to ballistic transport was clearly observed in vertical $\mathrm{Si}$ transistors [4].

Single-electron memory effects were studied using a short-channel MOSFET having Si quantum dots as a floating gate [9-13]. Storing of electrons in individual $\mathrm{Si}$ dots was evaluated by Kelvin probe force microscopy (KFM) [14]. We proposed novel memory devices based on nano electro-mechanical systems [15].

Photoluminescence and electron emission were observed from surface-oxidized nc-Si particles. Red light emission, observed at room temperature, was divided into two components. Efficiency of the no-phonon-assisted band enhanced and emission peak energy shifted toward high 
energy with decreasing core Si size [16]. Electron emission efficiency from nc-Si-based cold emitter device was as high as $5 \%$ [17].

We propose a novel functional material NeoSilicon, in which both quantum dot size and interparticle distance are precisely controlled. New functions in electron transport, photon emission, and electron emission are expected due to quantum effect at room temperature and large interaction between dots. The bandgap is determined by the particle size. Conductivity is controlled by tunneling distance. The transport characteristics are also controlled by charge quantization effect [18]. Technical challenges for implementation of NeoSilicon include precise control of quantum dot size, barrier layer and dot position. A particle size of $3-5 \mathrm{~nm}$, where energy quantization and charge quantization becomes significant at room temperature is required. A barrier layer of 1-2 nm thickness without interface traps is essential for electron tunneling. Dot position control provides additional merit for some applications such as quantum cellular automata. Assembly of high-density array of nc-Si dots was studied using drop and evaporation method of particle-dispersed solution.

\section{Silicon nanocrystal dot memory}

Nonvolatile memory devices using nc-Si-dot-based electrically isolated charge-storage nodes in an oxide film as a floating gate have emerged in the last decade [2], where charge leakages through localized oxide defects could be greatly reduced, therefore, improving performance reliabilities as well as the memory-retention time. Furthermore, a much thinner tunneling oxide film in nanodot memory devices causes a major tunneling mechanism due to direct tunneling hence enabling a faster write/erase speed compared to Fowler-Nordheim tunneling which is met in conventional flash type memory devices. Charge injection and storage in dense arrays of nc-Si dots in $\mathrm{SiO}_{2}$ is a key aspect of their performance. The ultimate goal for this class of devices is approaching the few- or single-electron storage in a small number of nc-Si elements at room temperature, relying on the Coulomb blockade effects as a new electron transport principle, commonly referred to as singleelectron memory. The high-density integration capability and low-power consumption enable them to be an attractive candidate for the next generation digital nonvolatile memory applications. However, in spite of the successful demonstrations of memory operations based on single-electron transports, the charge retention time of nc$\mathrm{Si}$ dot memory devices is too short for practical nonvolatile memory applications [10]. The improved retention time was demonstrated to be possible without any significant loss of programming speed based on the modifications of floating-gates by the dual memory nodes [11].

Charge retention characteristics reveal how charges are stored in nc-Si memory nodes. A major essential issue for nc-Si memory devices in nonvolatile memory applications is the data retention characteristics over extended period of time. After some electrons are trapped, at a chosen reading voltage (e.g., flat-band voltage), the stored electrons have a finite probability to tunnel back to the drain, which could cause a gradual shift of channel current or capacitance of $\mathrm{SiO}_{2} / \mathrm{nc}-\mathrm{Si} / \mathrm{SiO}_{2}$ diode. These gradual shifts reflect barrier heights/widths, internal electric fields, defect or interfacial states, and so on. Therefore, investigations of time dependences of memory windows as well as charging/ discharging behaviors of $\mathrm{nc}-\mathrm{Si}$ dots correlated with Coulomb blockade and quantum confinement effects offer better understanding of charge retention characteristics of nc-Si memory devices [12].

Investigation of charging and discharging in $\mathrm{nc}-\mathrm{Si}$ dots, based on measurements of capacitance-voltage and conductance-voltage characteristics, shows that interface

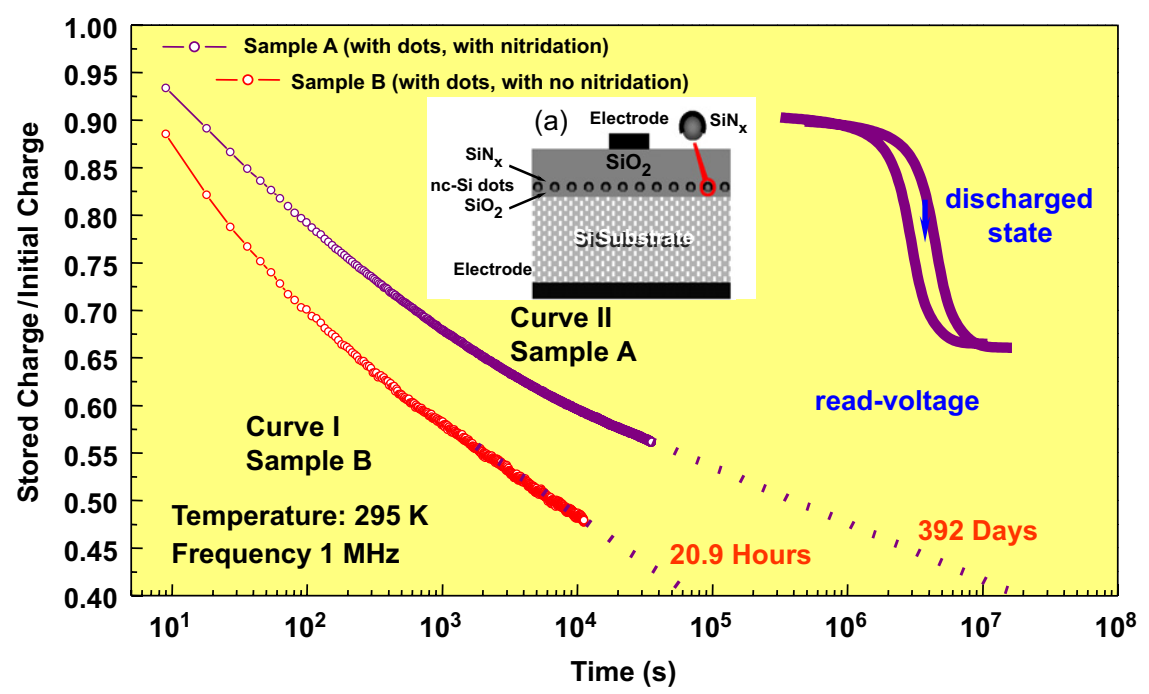

Fig. 1. Time dependence of the stored-charge from the flat-band state, after electrons were injected in floating-gates, shows a logarithmic discharging behavior. The gate voltage was kept at the initial flat-band voltage: $-1.6 \mathrm{~V}$ for sample A (surface nitrided nc-Si dots) and $-2.4 \mathrm{~V}$ for sample B (without nitridation). The inset shows the structure of memory device. 
a

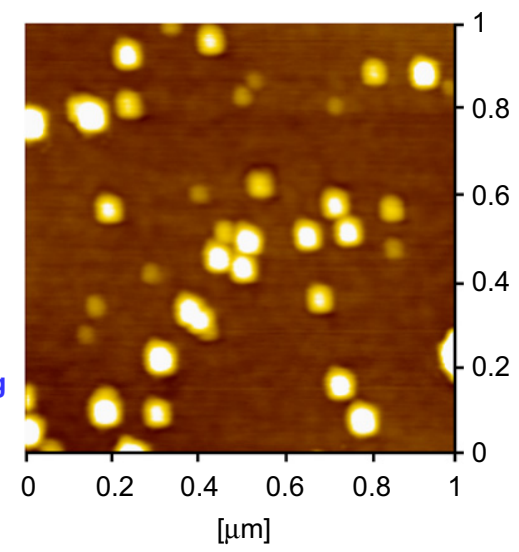

$$
0.00
$$

9.36

[nm]

Topography

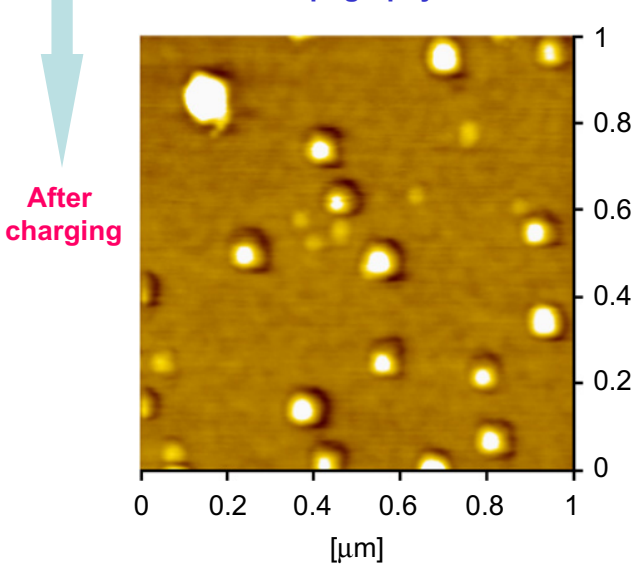

0.00

8.59

[nm]

Topography

b

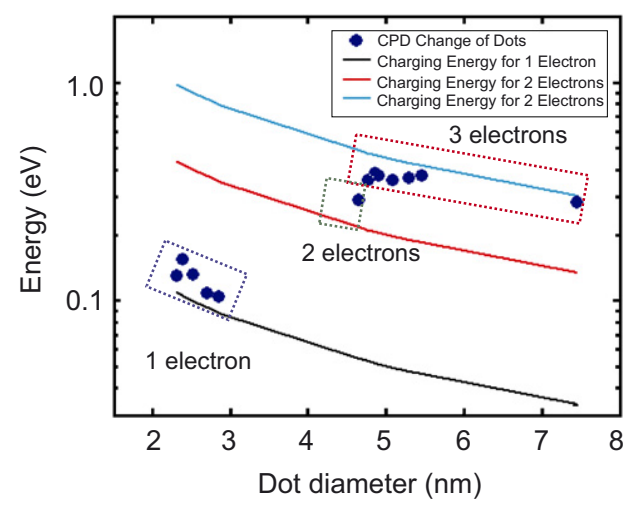

5 minutes after charging

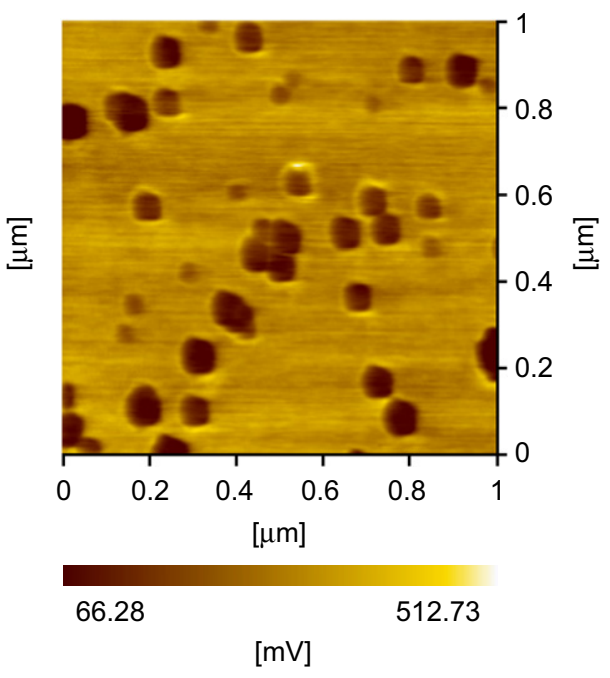

Potential

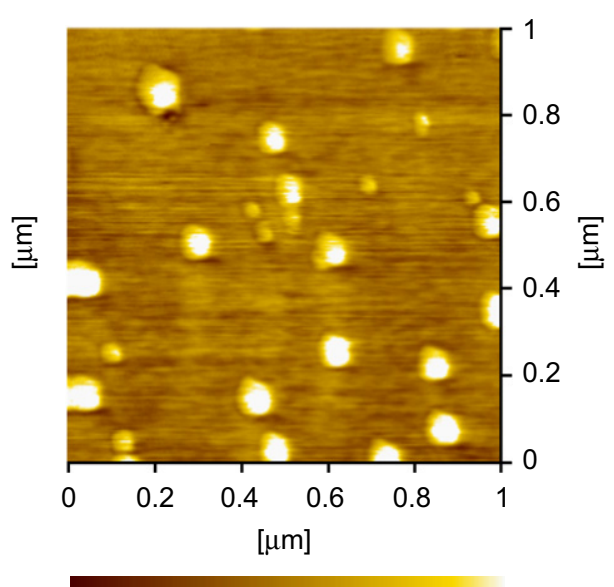

156.66

429.70

[mV]

Potential

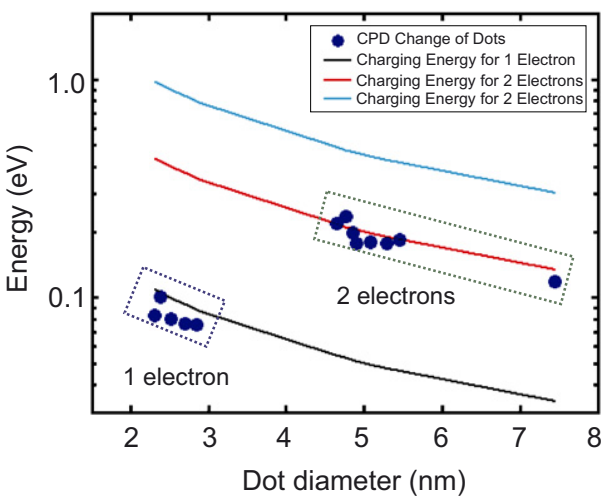

75 minutes after charging

Fig. 2. (a) Simultaneous observation of topography and potential images for nc-Si dots using non-contact AFM/KFM. For this measurement, nc-Si dots with various sizes are prepared deliberately so that the size dependence of potential can be evaluated by a single measurement. Dot size can be measured by the height of AFM measurements. (b) The change in the contact potential difference (CPD) observed for the nc-Si dots with various values of dot diameter (solid circles) and the calculated charging energy as a function of the dot diameter with the number of extra electrons on the dot of one, two and three. 
states and deep defects correspond to charging and discharging processes. However, at low defect and interfacial state density, electron charge/discharge is only dominated by bounding electrons in nc-Si dots. For a $\mathrm{SiO}_{2} / \mathrm{nc}-\mathrm{Si} / \mathrm{SiO}_{2}$ capacitor memory device, in time-dependent capacitance measurements, the memory-retention time was found to exceed $5 \mathrm{~h}$ (calculated time for the loss of about $10 \%$ of the original charge) at room temperature. Instead of being governed by deep defects, at low defect and interfacial state density, electron charge/discharge is only dominated by electron bounded in nc-Si dots. A repulsive "built-in" electric field from nc-Si dots to silicon substrate created and controlled by charge loss in nc-Si dots was proposed to explain such long-term retention time [13]. On the other hand, a longer retention time can be achieved by introducing a certain number of deep trapping centers in nc-Si dots with decreasing the interfacial states at the tunneling-oxide/Si interface. Compared with pure nc-Si floating-gate memory devices, nitrided nc-Si-dot-based memory devices were experimentally demonstrated to be helpful to remarkably increase retention time by three orders of magnitude, as shown in Fig. 1, without sacrificing write/erase time, in which memory operations based on combined charge/discharge processes of nitrided nc-Si dot systems. The stored charges in such memory nodes were identified not only in nc-Si dots but also in defect-states of silicon-nitride films, corresponding to electron delocalized and localized states, respectively.

\section{Charge storage study in silicon dots by KFM}

Charge transport study in nc-Si dots has attracted considerable interest along with the continuous development of quantum nano devices. Although traditional $I-V$ and $C-V$ studies provide vast amount of information about macroscopic device behavior, atomic force microscopy (AFM), on the other hand, is the suitable choice for studying microscopic nature of charge transport through nc-Si dots.

Counting the number of electrons stored in individual nc-Si dots is established using the AFM-based KFM. As shown in Fig. 2(a), the size of nc-Si dots can be evaluated precisely by the height of AFM measurement. Identifying dots containing single or few electrons is made possible using simple comparison between their potential change upon charging and the charging energies corresponding to the same number of electrons. As shown in Fig. 2(b), this approach not only permits the evaluation of stored electrons in individual dots at normal ambient conditions, but also enables the direct monitoring of dynamic characteristics of charge decay [14].

\section{Silicon photonic devices}

The discovery of optical gain in nc-Si reported by Pavesi [19] triggered widespread research for silicon-based lasers. Stimulated emission was reported for a nanostructured silicon pn junction diode using current injection [20]. Silicon-based Raman lasers have been demonstrated with pulsed optical pumping [21] and continuous wave operation [22].

Silicon-based laser operation is expected as a key technology in realizing opto-electronic integrated circuits which circumvent the problem of interconnect bottleneck in the state-of-the-art ULSI technology. However, siliconbased lasers operate only under very high excitation conditions [20-22], making it difficult to incorporate them into the CMOS circuits in which ultra low power consumptions are required.

We propose a completely different approach to realize silicon-based laser operation using three-dimensional photonic crystal structures combined with nc-Si quantum dots as light and electron emitters, as shown in Fig. 3. First, the three-dimensional photonic crystal structures are introduced to increase the stimulated emission probability caused by the standing wave at the photonic band edge, thereby resulting in a significant increase in the external quantum efficiency. Second, nc-Si quantum dots are used for constructing an active layer which emits light in the visible band due to the quantum confinement effect. As for
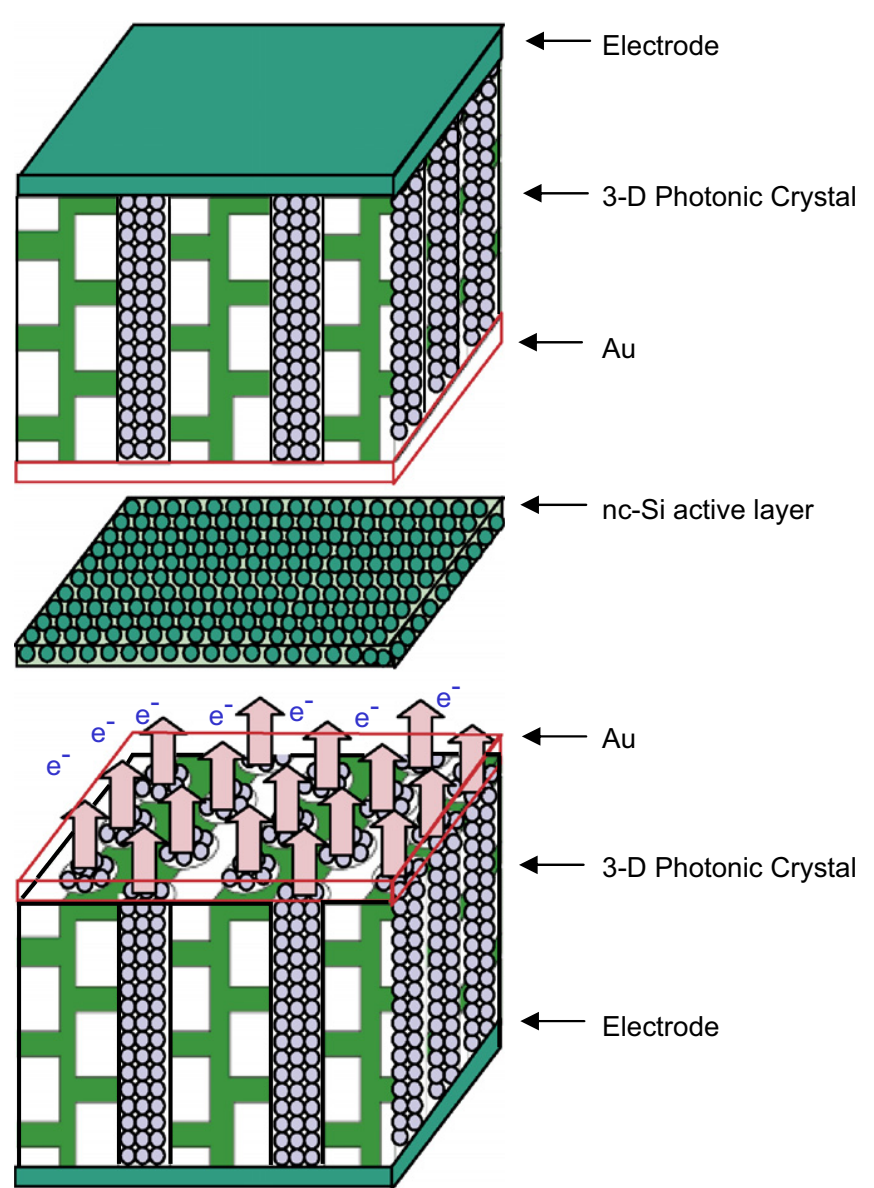

Fig. 3. Structure of all silicon-based light emitting device, which consists of nc-Si active layer with quasi-direct radiative recombination, nc-Si highefficiency electron emitter for excitation, and three-dimensional photonic crystal layer for optical confinement. 
the excitation mechanism, current injection is preferred for any practical applications. However, there has so far been no report on optical gain from nc-Si under current injection although high efficiency band-edge electroluminescence has already been reported for silicon light emitting diodes. One of the reasons is that current injection into nc-Si causes a significant decrease in the internal quantum efficiency due to the interfacial oxide layer. To circumvent this problem, we propose a new high-energy electron emission approach which is unique to nc-Si dot array structures [17,23].

Our nc-Si light emitting device features the electron emission structure based on $\mathrm{nc}-\mathrm{Si}$ arrays embedded in three-dimensional photonic crystal. Nanocrystalline silicon was fabricated with uniform diameter of $8 \mathrm{~nm}$ using pulsed gas plasma CVD method. A high-efficiency visible photoluminescence was observed for the surface-oxidized nc-Si indicating the quantum confinement effect and quasi-direct recombination [16]. Another key feature of our device is that the dimension of nc-Si can be controlled simply by changing the thermal oxidation time [24]. This is vital to control the electron emission properties, the luminescence wavelength of nc-Si, the refractive index of the structured nc-Si arrays, and therefore to optimize the photonic bandgap properties of the whole device.

\section{Conclusions}

Silicon nanocrystals with novel functions such as charge and energy quantization and efficient photon/electron emission are promising for future electronics applications. Silicon nanodot memory device can be a promising alternative for high-density nonvolatile memory. Microscopic nature of charge storage can be observed by KFM measurements. Light emitting devices embedded in Si chips can solve the problem of global interconnections in ULSI.

\section{Acknowledgments}

The authors thank former and current laboratory members, particularly M. Otobe, A. Dutta, K. Nishiguchi, B.J. Hinds, K. Arai, A. Tanaka, K. Usami, and Y.
Tsuchiya for experiments and discussion. This work was supported by CREST and SORST from Japan Science and Technology Agency (JST).

\section{References}

[1] S. Oda, D. Ferry (Eds.), Silicon Nanoelectronics, Taylor \& Francis, Boca Raton, 2006.

[2] J.J. Welser, S. Tiwari, S. Rishton, K.Y. Lee, Y. Lee, IEEE Electron. Devices Lett. 18 (1997) 278.

[3] K. Nishiguchi, S. Oda, J. Appl. Phys. 92 (3) (2002) 1399.

[4] K.D. Hirschman, L. Tsybeskov, S.P. Duttagupta, P.M. Fauchet, Nature 384 (1996) 338.

[5] N. Koshida, X. Sheng, T. Komada, Appl. Surf. Sci. 146 (1999) 371.

[6] T. Ifuku, M. Otobe, A. Itoh, S. Oda, Jpn. J. Appl. Phys. 36 (1997) 4031.

[7] A. Dutta, S. Oda, Y. Fu, M. Willander, Jpn. J. Appl. Phys. 39 (7B) (2000) 4647.

[8] K. Nishiguchi, S. Oda, J. Appl. Phys. 88 (7) (2000) 4186.

[9] A. Dutta, Y. Hayafune, S. Oda, Jpn. J. Appl. Phys. 39 (8B) (2000) L855.

[10] B.J. Hinds, T. Yamanaka, S. Oda, J. Appl. Phys. 90 (12) (2001) 6402.

[11] S. Huang, K. Arai, K. Usami, S. Oda, IEEE Trans. Nanotechnol. 3 (2004) 210.

[12] S. Huang, R.T. Tung, S. Oda, J. Appl. Phys. 94 (2003) 7261.

[13] S. Huang, S. Banerjee, R.T. Tung, S. Oda, J. Appl. Phys. 93 (2003) 7261.

[14] M.A. Salem, H. Mizuta, S. Oda, Appl. Phys. Lett. 85 (15) (2004) 3262.

[15] Y. Tsuchiya, K. Takai, N. Momo, S. Yamaguchi, T. Shimada, S. Koyama, K. Takashima, Y. Higo, H. Mizuta, S. Oda, IEEE Silicon Nanoelectronics Workshop, Honolulu, 2004.

[16] K. Arai, J. Omachi, K. Nishiguchi, S. Oda, Mater. Res. Soc. Symp. Proc. 664 (2001) A20.6.

[17] K. Nishiguchi, X. Zhao, S. Oda, J. Appl. Phys. 92 (5) (2002) 2748.

[18] S. Oda, Mater. Sci. Eng., B 101 (2003) 19.

[19] L. Pavesi, L. Dal Negro, C. Mazzoleni, G. Franzo, F. Priolo, Nature 408 (2000) 440.

[20] M.J. Chen, J.L. Yen, J.Y. Li, J.F. Chang, S.C. Tsai, C.S. Tsai, Appl. Phys. Lett. 84 (2004) 2163.

[21] O. Boyraz, B. Jalali, Opt. Express 12 (2004) 5269.

[22] H. Rong, R. Jones, A. Liu, O. Cohen, D. Hak, A. Fang, M. Paniccia, Nature 433 (2005) 725.

[23] S. Uno, K. Nakazato, S. Yamaguchi, A. Kojima, N. Koshida, H. Mizuta, IEEE Trans. Nanotechnol. 2 (4) (2003) 301.

[24] J. Omachi, R. Nakamura, K. Nishiguchi, S. Oda, Mater. Res. Soc. Symp. Proc. 638 (2001) F5. 3. 1. 